\title{
Anisotropic Babinet-invertible metasurfaces to realize transmission-reflection switching for orthogonal polarizations of light
}

\author{
Yosuke Nakata, , , - Yoshiro Urade, ${ }^{2}$ Kunio Okimura, ${ }^{3}$ Toshihiro Nakanishi, ${ }^{2}$ \\ Fumiaki Miyamaru, ${ }^{1,4}$ Mitsuo Wada Takeda, ${ }^{4}$ and Masao Kitano ${ }^{2}$ \\ ${ }^{1}$ Center for Energy and Environmental Science, Shinshu University, 4-17-1 Wakasato, Nagano 380-8553, Japan \\ ${ }^{2}$ Department of Electronic Science and Engineering, Kyoto University, Kyoto 615-8510, Japan \\ ${ }^{3}$ School of Engineering, Tokai University, 4-1-1 Kitakaname, Hiratsuka, Kanagawa 259-1292, Japan \\ ${ }^{4}$ Department of Physics, Faculty of Science, Shinshu University, \\ 3-1-1 Asahi, Matsumoto, Nagano 390-8621, Japan
}

(Dated: Compiled September 12, 2018)

\begin{abstract}
The electromagnetic properties of an extremely thin metallic checkerboard drastically change from resonant reflection (transmission) to resonant transmission (reflection) when the local electrical conductivity at the interconnection points of the checkerboard is switched. To date, such critical transitions of metasurfaces have been applied only when they have 4-fold rotational symmetry, and their application to polarization control, which requires anisotropy, has been unexplored. To overcome this applicability limitation and open up new pathways for dynamic deep-subwavelength polarization control by utilizing critical transitions of checkerboard-like metasurfaces, we introduce a universal class of anisotropic Babinet-invertible metasurfaces enabling transmission-reflection switching for each orthogonally polarized wave. As an application of anisotropic Babinet-invertible metasurfaces, we experimentally realize a reconfigurable terahertz polarizer whose transmitting axis can be dynamically rotated by $90^{\circ}$.
\end{abstract}

\section{INTRODUCTION}

Metamaterials, which are artificial materials composed of engineered structures, exhibit exotic functionality not readily observed in nature [1]. Two-dimensional metamaterials with thicknesses much thinner than the wavelength of light, are called metasurfaces [2]. The careful design of metasurfaces enables us to control both the amplitude and phase of electromagnetic waves [3, 4]. Due to the scale invariance of Maxwell equations, the extraordinary properties of metamaterials and metasurfaces can be theoretically realized for all frequency ranges by altering the size of the structures. In the terahertz frequency range, metamaterials and metasurfaces are considered promising candidates for manipulating terahertz waves, because conventional electronics and photonics technologies cannot be directly applied. Various passive and active devices based on metamaterials and metasurfaces have been demonstrated in the terahertz frequency range [5].

When designing metasurfaces in a vacuum, Babinet's principle is often utilized. Babinet's principle relates the scattering fields of a metasurface to a complementary metasurface. The complementary metasurface is obtained by applying a structural inversion to interchange the holes and metallic portions of the given one-layer metallic metasurface [6]. For a more general case, consider a metasurface at $z=0$ with a spatially varying sheet impedance of $Z_{\mathrm{s}}(x, y)$, which relates the tangential component (namely, $x y$-component) of the electric field at $(x, y)$ on $z=0$ and the surface current den-

\footnotetext{
* y_nakata@shinshu-u.ac.jp
}

sity at the same point [7]. The complementary surface with sheet impedance $Z_{\mathrm{s}}^{\text {(comp) }}(x, y)$ is obtained by applying a impedance inversion defined as $Z_{\mathrm{s}}^{(\text {comp })}(x, y)=$ $Z_{0}^{2} /\left(4 Z_{\mathrm{s}}(x, y)\right)$, where $Z_{0} \sim 377 \Omega$ is the impedance of the vacuum [8].

(a)
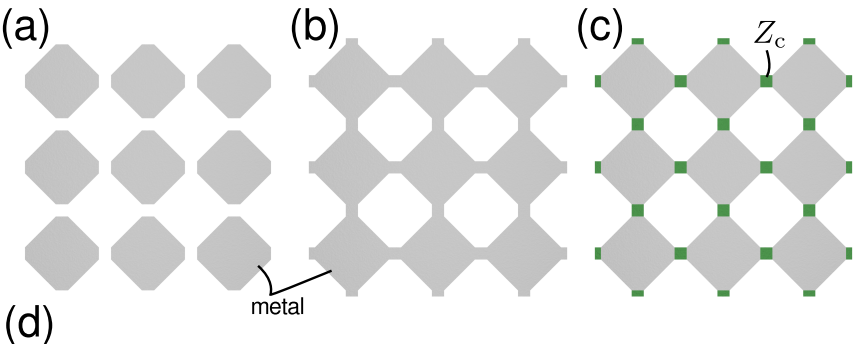

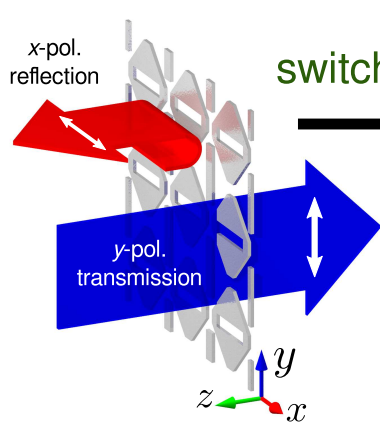

off state
FIG. 1. (a) Off-state checkerboard metasurface. (b) On-state checkerboard metasurface. (c) Babinet-invertible checkerboard metasurface with variable-resistance sheet with sheet impedance $Z_{\mathrm{c}}$. (d) Dynamic rotation of the transmitting axis of reconfigurable polarizer. The anisotropic checkerboard can be utilized to realize this functionality. 
When considering Babinet's principle, the metallic checkerboard is a special system: the disconnected [offstate; Fig. 11(a)] and connected [on-state; Fig. 11(b)] checkerboards are complementary with each other [9]. Their electromagnetic scattering properties drastically change when the connectivity of the checkerboard is altered. Such a critical behavior of checkerboard metasurfaces has been theoretically investigated from the perspective of percolation theory [10] and experimentally observed in the microwave 11] and terahertz frequency ranges [9, 12]. The checkerboard metasurface has also been characterized in the optical region [13]. The critical property of checkerboard metasurfaces has been applied to reconfigurable transmission lines [14, 15] and recently the critical behavior caused by rotational disorder has also been investigated [16].

To control the criticality of the checkerboard, resistive sheets with sheet impedance $Z_{\mathrm{c}}$ can be introduced at the interconnection points of the checkerboard, as shown in Fig. 1(c) [17 19]. The reconfigurable checkerboard is a checkerboard metasurface with dynamically controllable $Z_{\text {c }}$. If a metasurface is dynamically switchable to the metasurface congruent to the complement of the original, like the reconfigurable checkerboard, it is called Babinet invertible. Babinet-invertible metasurfaces are considered to be a generalization of the reconfigurable checkerboard. The 4-fold rotational symmetry of the reconfigurable checkerboard and Babinet's principle lead to the relation

$$
\tilde{t}^{(\text {off })}(\omega)+\tilde{t}^{(\text {on })}(\omega)=1,
$$

where the (zeroth-order) complex amplitude transmission coefficients of a normally incident plane wave with an angular frequency $\omega$ are denoted by $\tilde{t}^{(\text {off })}(\omega)$ and $\tilde{t}^{(\text {on })}(\omega)$ for the off-state $\left(Z_{\mathrm{c}}=\infty\right)$ and on-state $\left(Z_{\mathrm{c}}=0\right)$ metasurfaces, respectively [20]. Then, the complementary switching of the reconfigurable checkerboard realizes transmission inversion, represented by Eq. (1). The transmission inversion of the reconfigurable checkerboard has been applied to a reconfigurable capacitive-inductive terahertz filter [21].

In previous studies of Babinet-invertible metasurfaces, 4-fold symmetry is assumed and the polarization dependence of the electromagnetic properties vanishes for normal incidence. Thus, the possibility of dynamic polarization control, which is essential for polarization-sensitive spectroscopy [22, 23], through complementary switching has been unexplored. In this paper, the applicability limitation of the critical transition of checkerboard metasurfaces to dynamic deep-subwavelength polarization control is overcome by introducing a universal class of anisotropic Babinet-invertible metasurfaces enabling transmission inversions for each orthogonally polarized wave. To demonstrate one potential application of the proposed class of metasurfaces, we experimentally realize a reconfigurable terahertz polarizer as an anisotropic Babinet-invertible metasurface.

\section{THEORY}

If we break the rotational symmetry of Babinetinvertible metasurfaces, the (zeroth-order) complex amplitude transmission coefficients $\tilde{t}_{x}$ and $\tilde{t}_{y}$ for $x$ - and $y$ polarized incident waves must be distinguished. Here, we consider an extension of Eq. (11) to such an anisotropic metasurface as follows:

$$
\begin{aligned}
& \tilde{t}_{x}^{\text {(off })}(\omega)+\tilde{t}_{x}^{\text {(on })}(\omega)=1 \\
& \tilde{t}_{y}^{\text {(off })}(\omega)+\tilde{t}_{y}^{\text {(on })}(\omega)=1 .
\end{aligned}
$$

Equations (2) and (3) express transmission inversion separately for the two axes $x$ and $y$. We therefore name this inversion transmission inversion for each orthogonal polarization, which requires a specific symmetry. Note that transmission inversion for each orthogonal polarization and the electric field continuation condition $1+\tilde{r}_{i}^{\text {(off) }}(\omega)=\tilde{t}_{i}^{\text {(off) }}(\omega)$ lead to transmission-reflection switching for each orthogonal polarization represented by

$$
\tilde{t}_{i}^{\text {(on })}(\omega)=-\tilde{r}_{i}^{(\text {off })}(\omega),
$$

where $\tilde{r}_{i}^{(\text {off })}(\omega)$ is a complex amplitude reflection coefficient for an $i$-polarized normally incident plane wave.

Next, we discuss the sufficient symmetry to realize transmission inversion for each orthogonal polarization. The rigorous vector version of Babinet's principle is expressed as follows [17]:

$$
\begin{aligned}
& \tilde{t}_{x}^{\text {(off })}(\omega)+\tilde{t}_{y}^{(\mathrm{comp})}(\omega)=1, \\
& \tilde{t}_{y}^{\text {(off })}(\omega)+\tilde{t}_{x}^{(\mathrm{comp})}(\omega)=1,
\end{aligned}
$$

where $\tilde{t}_{i}^{\text {(comp) }}(\omega)$ represents the (zeroth-order) complex amplitude transmission coefficient for a normally incident plane wave with $i$-polarization onto the metasurface complementary to the off-state one $(i=x, y)$. Note that the polarization is rotated by $90^{\circ}$ in the complementary problem compared with the original. Comparing Eqs. (5)(6) with Eqs. (2)-(3), transmission inversion for each orthogonal polarization requires $\tilde{t}_{x}^{(\text {on })}(\omega)=\tilde{t}_{y}^{(\operatorname{comp})}(\omega)$ and $\tilde{t}_{y}^{\text {(on) }}(\omega)=\tilde{t}_{x}^{\text {(comp) }}(\omega)$. These equations are satisfied when the metasurface complementary to the off-state surface is obtained by $90^{\circ}$ rotation of the on-state surface. This is the sufficient symmetry condition to realize transmission inversion for each orthogonal polarization.

As an application of transmission inversion for each orthogonal polarization, we consider the design of a polarizer whose transmitting axis can be dynamically rotated by $90^{\circ}$, as shown in Fig. 1(d). Designing such a reconfigurable polarizer with variable-resistance elements requires simultaneous fine tuning for both the onand off-state metasurfaces. This is difficult because the on- and off-state metasurfaces generally depend on each other. However, by utilizing transmission inversion for each orthogonal polarization, the problem can be drastically simplified. If we have an off-state Babinet-invertible 

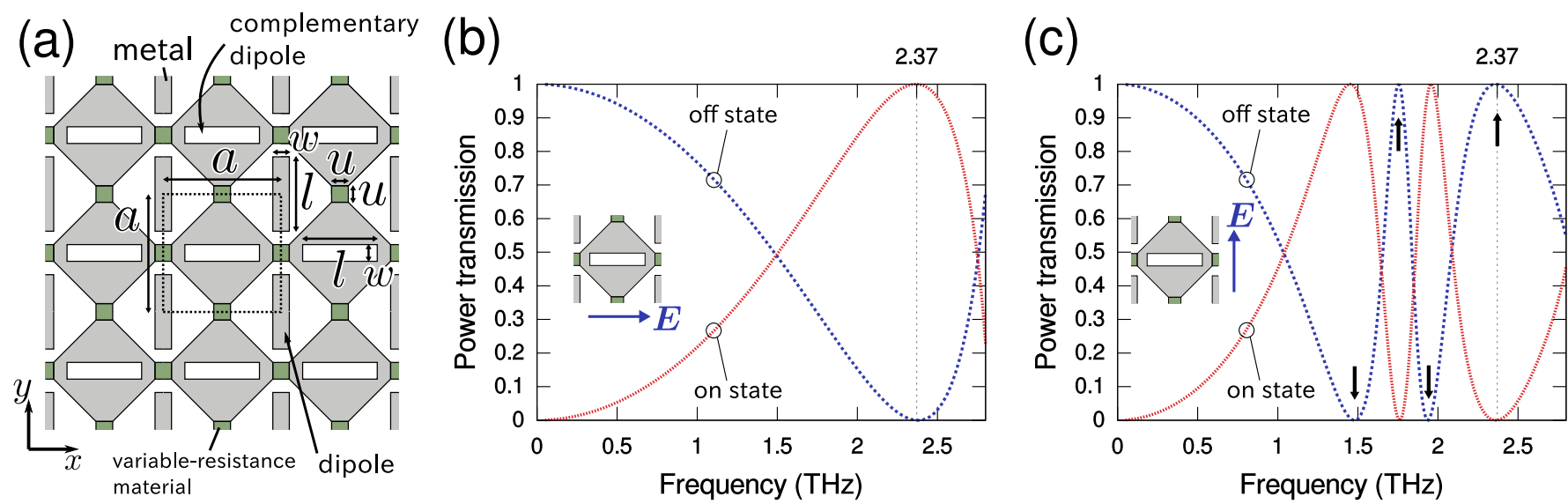

FIG. 2. (a) Design of dipole-nested Babinet-invertible checkerboard metasurface (D-checkerboard) with $a=106 \mu$ m, $u=w=$ $15 \mu \mathrm{m}$, and $l=67 \mu \mathrm{m}$. Simulated power transmission spectra of ideal on- and off-state D-checkerboards at the normal incidence of (b) $x$ - and (c) $y$-polarized plane waves.

metasurface with $\tilde{t}_{x}^{\text {(off) }}\left(\omega_{0}\right) \sim 0$ and $\tilde{t}_{y}^{\text {(off })}\left(\omega_{0}\right) \sim 1$ at an angular frequency $\omega_{0}$, then $\tilde{t}_{x}^{\text {(on })}\left(\omega_{0}\right) \sim 1$ and $\tilde{t}_{y}^{(\text {on })}\left(\omega_{0}\right) \sim$ 0 are automatically satisfied for the on-state metasurface under transmission inversion for each orthogonal polarization. Then, the transmitting axis of the polarizer can be dynamically rotated by $90^{\circ}$. The problem is reduced to designing the off-state Babinet-invertible metasurface with $\tilde{t}_{x}^{\text {(off) }}\left(\omega_{0}\right) \sim 0$ and $\tilde{t}_{y}^{\text {(off })}\left(\omega_{0}\right) \sim 1$.

We design the reconfigurable terahertz polarizer by using the finite element method solver (COMSOL MULTIPHYSICS). To break the 4-fold symmetry of the checkerboard, we nest dipoles with their complementary pairs into the checkerboard so that the sufficient condition to realize transmission inversion for each orthogonal polarization can be satisfied. The design of a dipolenested Babinet-invertible checkerboard metasurface (Dcheckerboard) on $z=0$ is shown in Fig. 2(a). To simplify the analysis, we assume that all the metal sections are zero-thickness perfect electric conductors. The calculations are performed in the unit cell and periodic boundary conditions are applied to the sides. The top and bottom boundaries are set as ports with only fundamental modes. The plane wave generated by the top port normally enters a metasurface from $z>0$ to $z<0$. The basic parameters are set as $a=106 \mu \mathrm{m}$ and $u=w=15 \mu \mathrm{m}$. The dipoles and their complements respond only for $y$ polarization. For $x$-polarized normally incident waves, the structure responds like a checkerboard without the dipoles. To realize $\tilde{t}_{x}^{\text {(off) }}\left(\omega_{0}\right) \sim 0$, we set a target angular frequency of $\omega_{0} \sim 2 \pi \times 2.4 \mathrm{THz}$ near the resonant frequency of the checkerboard. Tuning the dipole length $l$ to realize $\tilde{t}_{y}^{\text {(off) }}\left(\omega_{0}\right) \sim 1$, we obtain $l=67 \mu \mathrm{m}$. Figures 2 (b) and (c) show the calculated power transmission spectra of off- and on-state D-checkerboards. We observe a $90^{\circ}$ transmitting-axis rotation around $\omega=\omega_{0}$. Due to the resonance nature at $\omega=\omega_{0}$, an extremely high extinction ratio is achieved in spite of the deep-subwavelength thickness of the metasurface. We also observe that the power

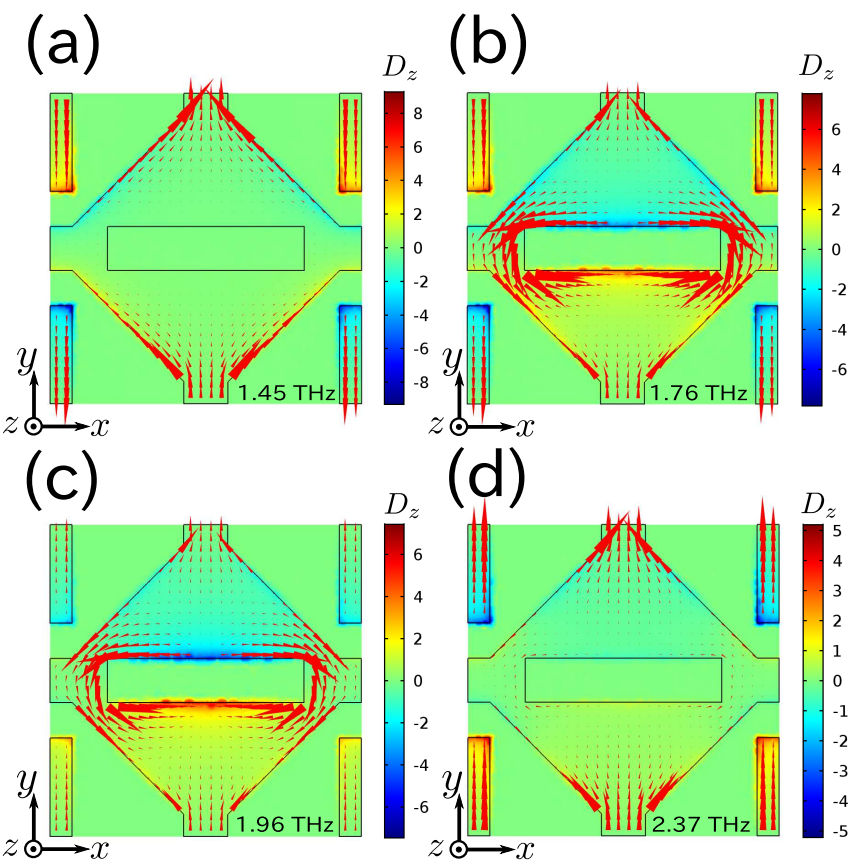

FIG. 3. Electric displacement $D_{z}$ on the plane $z=a / 1000$, corresponding to the surface charge density, and surface current density of the ideal on-state D-checkerboard at (a) $\omega /(2 \pi)=1.45 \mathrm{THz}$, (b) $1.76 \mathrm{THz}$, (c) $1.96 \mathrm{THz}$, and (d) $2.37 \mathrm{THz}$ for $y$-polarized plane waves normally incident from $z>0$ to $z<0$. The phase of $D_{z}$ is shifted by $90^{\circ}$ compared with the surface current.

transmission spectra are accurately inverted with respect to power transmission $T=1 / 2$ under the switching. This phenomenon can be explained by Eq. (4). Due to the mirror symmetry with respect to the $x$ and $y$ axes for the D-checkerboard, conversion between $x$ and $y$ polarizations does not occur. Then, the power conservation law gives $\left|\tilde{t}_{i}^{\text {(off) }}(\omega)\right|^{2}+\left|\tilde{r}_{i}^{\text {off })}(\omega)\right|^{2}=1$ at the angular frequency $\omega \leq \omega_{\mathrm{d}}=2 \pi c / a=2 \pi \times 2.83 \mathrm{THz}$ without any diffraction 

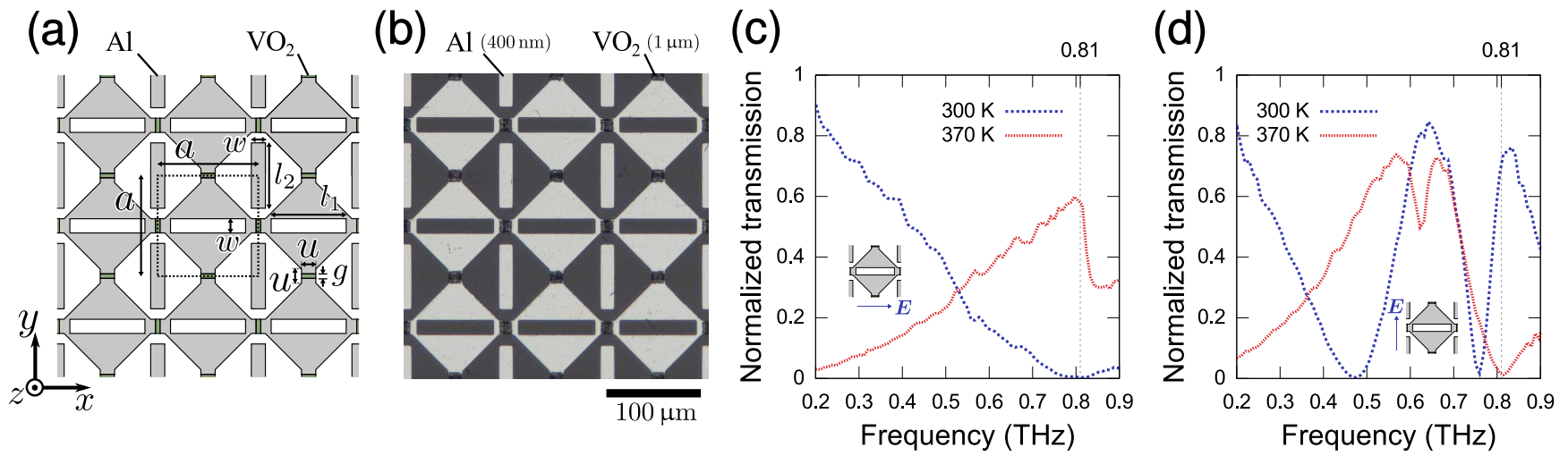

FIG. 4. (a) Design of a dipole-nested checkerboard metasurface on a sapphire substrate. (b) Micrograph of the fabricated D-checkerboard on a sapphire substrate with the target lengths $a=106 \mu \mathrm{m}, u=w=15 \mu \mathrm{m}, g=5 \mu \mathrm{m}, l_{1}=80 \mu \mathrm{m}$, and $l_{2}=69 \mu \mathrm{m}$. The thicknesses of $\mathrm{VO}_{2}$ and $\mathrm{Al}$ are $1 \mu \mathrm{m}$ and $400 \mathrm{~nm}$, respectively. Normalized power transmission spectra of the fabricated D-checkerboard for normal incidence of a collimated terahertz beam with (c) $x$ and (d) $y$ polarization.

to higher-order modes, where $c$ is the speed of light in a vacuum. Using Eq. (4), $\left|\tilde{t}_{i}^{\text {(off) }}(\omega)\right|^{2}+\left|\tilde{r}_{i}^{\text {(off) }}(\omega)\right|^{2}=1$ can be written as $\left|\tilde{t}_{i}^{\text {(off) }}(\omega)\right|^{2}+\left|\tilde{t}_{i}^{\text {on })}(\omega)\right|^{2}=1$. Figure 3 shows the charge and surface current density of the onstate D-checkerboard at $1.45 \mathrm{THz}, 1.76 \mathrm{THz}, 1.96 \mathrm{THz}$, and $2.37 \mathrm{THz}$ for $y$-polarized plane waves normally incident from $z>0$ to $z<0$. The field distributions at $1.45 \mathrm{THz}$ and $2.37 \mathrm{THz}$ correspond to those of the on-state D-checkerboard without complementary dipoles shown in Figs. 6(b) and (c) of the Appendix. These distributions are not greatly influenced by the introduction of complementary dipoles. On the other hand, the transmission dip at $1.76 \mathrm{THz}$ and peak at $1.96 \mathrm{THz}$ are caused by hybridization of the complementary dipole mode and those of the on-state D-checkerboards without complementary dipoles. The further detail of multimode formation in D-checkerboard is discussed in Appendix $\mathrm{A}$.

\section{EXPERIMENT}

Now, we consider the experimental demonstration of a reconfigurable D-checkerboard in the terahertz frequency range. For the variable-resistance sheets, we use vanadium dioxide $\left(\mathrm{VO}_{2}\right)$ on a c-cut sapphire substrate. As the temperature increases above $T_{\mathrm{c}} \approx 340 \mathrm{~K}, \mathrm{VO}_{2}$ exhibits an insulator-to-metal transition where the electrical conductivity typically changes by several orders of magnitude; thus, the metasurface transitions from the off state to the on state. Using the c-cut sapphire as a substrate at $z \leq 0$ causes mirror symmetry breaking with respect to $z=0$ where the structures are located, and the condition for Babinet's principle is no longer satisfied. In the target terahertz frequency range, c-cut sapphire has a refractive index $n_{x}=n_{y}=n_{\perp} \approx 3.1$ and $n_{z}=n_{\|} \approx 3.4$ [24]. To compensate this substrate effect, we must modify the design of the D-checkerboard, as shown in Fig. 4 (a). In the following discussion, we use the normalized transmission coefficient of the metasurface with a sapphire substrate as $\hat{t}_{i}=\tilde{t}_{i} / t_{0}$ for an $i$-polarized normally incident wave, where $\tilde{t}_{i}$ is the amplitude transmission coefficient of the plane $z=0$ with metasurface from air to the substrate, and $t_{0}=2 /\left(1+n_{\perp}\right)$ is the Fresnel coefficient for transmission from air to the sapphire. For $x$-polarized normally incident waves, we changed the gap $g=5 \mu \mathrm{m}$ to ensure $\hat{t}_{x}^{\text {(off) }}\left(\omega_{0}^{\prime}\right) \sim 0$ and $\hat{t}_{x}^{\text {on) }}\left(\omega_{0}^{\prime}\right) \sim 1$ [21], where $\omega_{0}^{\prime}$ is the modified resonant angular frequency. For $y$-polarized normally incident waves, we use different lengths for the dipoles and their complements. By optimizing these parameters, we obtain $l_{1}=80 \mu \mathrm{m}$ and $l_{2}=69 \mu \mathrm{m}$ to realize $\hat{t}_{y}^{\text {off })}\left(\omega_{0}^{\prime}\right) \sim 1$ and $\hat{t}_{y}^{\text {on })}\left(\omega_{0}^{\prime}\right) \sim 0$. The calculated transmission spectra of the designed metasurface are shown in Fig. 7 of the Appendix.

Next, we experimentally demonstrate dynamic rotation of the transmitting axis of the reconfigurable metasurface. The designed D-checkerboard is fabricated as follows. A thin film of stoichiometric $\mathrm{VO}_{2}$ of about 1$\mu \mathrm{m}$ thickness is deposited on a sapphire (0001) substrate (thickness: $1.0 \mathrm{~mm}$ ) via reactive magnetron sputtering of a vanadium target [25]. After a positive photoresist is patterned on the $\mathrm{VO}_{2}$ using a maskless lithography technique, the unnecessary part of the $\mathrm{VO}_{2}$ film is removed by wet etching. The D-checkerboard made of aluminum (thickness: $400 \mathrm{~nm}$ ) is formed by photolithography and electron beam evaporation at room temperature and liftoff techniques. A micrograph of the fabricated sample is shown in Fig. प(b). The $\mathrm{VO}_{2}$ squares with target side length $u$ are partially overlapped by the $\mathrm{Al}$ structures, for ensuring electrical connection. The maximum thickness of the device fabricated on a c-cut sapphire substrate is $\sim 1.4 \mu \mathrm{m}$, which is deep subwavelength compared with the wavelength at the target frequency.

To evaluate the D-checkerboard, we use a conventional terahertz time-domain spectroscopy system. To delay the signals reflected at the boundaries, two pieces of ccut sapphire substrates of thickness $1 \mathrm{~mm}$ are attached under the metasurface with a $1 \mathrm{~mm}$ c-cut sapphire sub- 
strate. Therefore, the overall thickness of the substrate is $3 \mathrm{~mm}$. A reference c-cut sapphire sample with thickness $3 \mathrm{~mm}$ is prepared by stacking three sapphire plates of thickness $1 \mathrm{~mm}$. The stacked samples are held by a brass holder with a temperature feedback system to control the electric current in a nichrome wire attached to the holder, while the temperature of the holder is monitored by a thermocouple. A collimated terahertz beam with a linear polarization and beam diameter $\sim 8 \mathrm{~mm}$ is normally incident onto the stacked samples. The temporal profile of the electric field is measured using a detector dipole antenna. The multiple reflected signal is cut using a time-domain window function. Measuring the electric fields $E_{\text {ref }}(t)$ of the c-cut reference sapphire substrate and $E(t)$, we calculate the normalized complex amplitude transmission coefficient $\hat{t}(\omega)=\tilde{E}(\omega) / \tilde{E}_{\text {ref }}(\omega)$, where $\tilde{E}(\omega)$ and $\tilde{E}_{\text {ref }}(\omega)$ are Fourier transformed electric fields. Figures 4(c) and (d) show the power transmission spectra $|\hat{t}|^{2}$ of the D-checkerboard on a sapphire substrate for normal incidence of collimated terahertz beams with $x$ and $y$ polarization, respectively. The lowest diffraction frequency of the D-checkerboard is given by $f_{\mathrm{d}}=c /\left(n_{\|} a\right) \sim 0.83 \mathrm{THz}$. At $0.81 \mathrm{THz}$, the dynamic rotation of the transmitting axis of the metasurface from the $y$ axis to the $x$ axis is realized by heating the device. The extinction ratios $\left|\hat{t}_{y}^{\text {(off) }}\left(\omega_{0}^{\prime}\right) / \hat{t}_{x}^{\text {(off })}\left(\omega_{0}^{\prime}\right)\right|^{2}$ and $\left|\hat{t}_{x}^{\text {on })}\left(\omega_{0}^{\prime}\right) / \hat{t}_{y}^{\text {on })}\left(\omega_{0}^{\prime}\right)\right|^{2}$ at $\omega_{0}^{\prime}=2 \pi \times 0.81 \mathrm{THz}$ are $\sim 10^{2}$. Although the frequency dependences of the transmission spectra agree well with the simulated data shown in Fig. 7 of the Appendix, the maximum transmission peaks of the on- and off-state metasurface are not as high as the simulated data. This is because the resistivity of the $\mathrm{VO}_{2}$ film is not switched ideally and the aluminum has a finite conductivity. High quality vanadium-dioxide films formed by other deposition techniques, such as pulse laser deposition [26], and higher conductive metals could improve the performance of the device.

\section{CONCLUSION}

In this paper, we introduced the class of anisotropic Babinet-invertible metasurfaces enabling transmissionreflection switching for each orthogonally polarized wave, and experimentally demonstrated their applicability to polarization control of electromagnetic waves. The transmission inversion for each orthogonal polarization can be considered as a manifestation of an artificially engineered insulator-metal transition of anisotropic metasurfaces. The concept of anisotropic Babinet-invertible metasurfaces enabling transmission-reflection switching for each orthogonally polarized wave is universal, and is independent of the implementation. Their applications are not limited to reconfigurable terahertz polarizers, but could find a wide range of applications for dynamic polarization control over a broad region of the electromagnetic spectrum in which the variable-resistance materials can be used. Ultrafast switching could be realized if photoexcitation of carriers is employed. Thus, the anisotropic Babinet-invertible metasurfaces will pave the way for ultra-fast polarization-selective spectroscopy.

\section{ACKNOWLEDGMENTS}

The authors gratefully acknowledge the contributions of T. Nishida and T. McArthur. The sample fabrication was performed with the help of Kyoto University Nano Technology Hub, as part of the "Nanotechnology Platform Project" sponsored by MEXT, Japan. The present research is supported by grant from the Murata Science Foundation and JSPS KAKENHI Grant Number JP16K13699. One of the authors (Y.U.) was supported by a JSPS Research Fellowship for Young Scientists.

\section{Appendix A: Multimode formation in D-checkerboard}

Here, we investigate the formation of the multiple resonances of an ideal D-checkerboard. The field distributions of on- and off-state metasurfaces are exactly related to each other, so we focus only on the on-state metasurface. We first study the transmission spectra and field distribution of each component of the ideal Dcheckerboard in a vacuum by COMSOL using the parameters for an ideal D-checkerboards shown in Fig. 2(b): $a=106 \mu \mathrm{m}, u=w=15 \mu \mathrm{m}$, and $l=67 \mu \mathrm{m}$. Periodic boundary conditions are applied at the sides of the unit cell, and ports with only lowest frequency modes are set on the bottom and top faces. The metallic structures are assumed to be zero-thickness perfect electric conductors. Normally incident $y$-polarized plane waves enter the metasurface at $z=0$ from $z>0$ to $z<0$. Figure 5)(a) shows the transmission spectra of the dipoles and the on-state checkerboard. The dipoles and the on-state checkerboard show a transmission dip at $2.03 \mathrm{THz}$ and transmission peak at $2.42 \mathrm{THz}$. The electric displacement $D_{z}$ just above the surface $(z=a / 1000)$, corresponding to the charge density, and the surface current distributions on the metal $(z=0)$ at these frequencies, are shown in Figs. 5(b) and (c). To clearly see the distributions, the phase of $D_{z}$ is shifted by $90^{\circ}$ compared with the surface current in these plots. The first-order resonance of the dipoles is shown in Fig. 5(b). In Fig. 5(c), the direction of the surface current at the center of the on-state checkerboard is opposite that on the top and bottom interconnection patches.

Next, we consider the on-state checkerboard combined with dipoles. The spectrum of the on-state checkerboard with dipoles at the normal incidence of $y$-polarized plane waves is shown in Fig. 6(a). It shows Fano-like characteristics [27] with transmission peaks at $1.45 \mathrm{THz}$ and $2.72 \mathrm{THz}$, and a transmission dip at $2.32 \mathrm{THz}$. The charge and surface current distribution on the dipoles at 
(a)

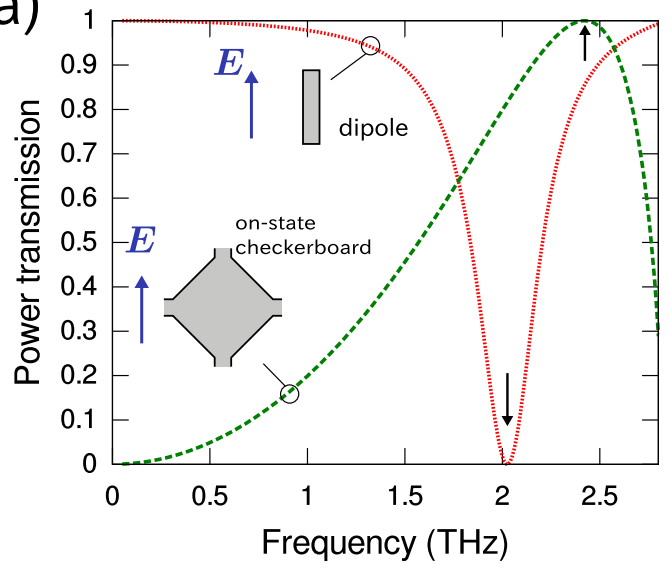

(b)

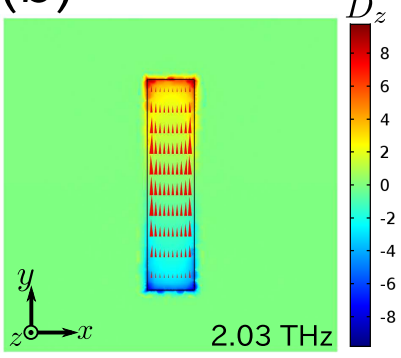

(c)

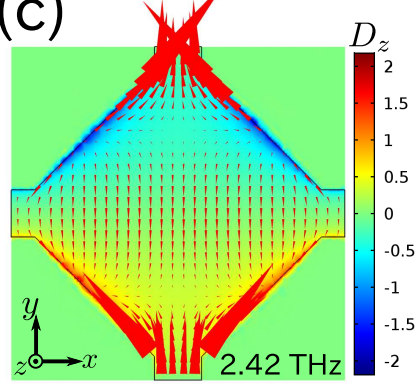

FIG. 5. (a) Transmission spectra of dipoles and the onstate checkerboard with $a=106 \mu \mathrm{m}, u=w=15 \mu \mathrm{m}$, and $l=67 \mu \mathrm{m}$. The electric displacement $D_{z}$ on the plane $z=a / 1000$, corresponding to the surface charge density, and surface current density of (b) dipoles at $\omega /(2 \pi)=2.03 \mathrm{THz}$ and (c) the on-state checkerboard at $2.42 \mathrm{THz}$. The phase of $D_{z}$ is shifted by $90^{\circ}$ compared with the surface current.

$1.45 \mathrm{THz}$ [Fig. 6(b)] and $2.32 \mathrm{THz}$ [Fig. 6(c)] are similar to those shown in Fig. 馬(b). At these frequencies, the phase relations between the currents on the checkerboard and the dipole are opposite, and the resonant transmission at $1.45 \mathrm{THz}$ and reflection at $2.32 \mathrm{THz}$ are caused by interference between the first-order dipole mode and the on-state checkerboard mode. The current distributions on the checkerboard and the dipole at $2.72 \mathrm{THz}$ in Fig. 6(d) are similar to those in Fig. 5(b) and (c), respectively. This indicates that the $2.72 \mathrm{THz}$ resonant transmission originates from a hybridized mode of the first-order dipole mode and $2.42 \mathrm{THz}$ on-state checkerboard mode.

\section{Appendix B: Simulation for modified D-checkerboard on a c-cut sapphire substrate}

Here, we present spectra of a modified D-checkerboard on a c-cut sapphire substrate. The geometrical parameters are $a=106 \mu \mathrm{m}, u=w=15 \mu \mathrm{m}, g=5 \mu \mathrm{m}$, $l_{1}=80 \mu \mathrm{m}$, and $l_{2}=69 \mu \mathrm{m}$. We assume that all conductive parts are zero-thickness perfect electric conduc- (a)

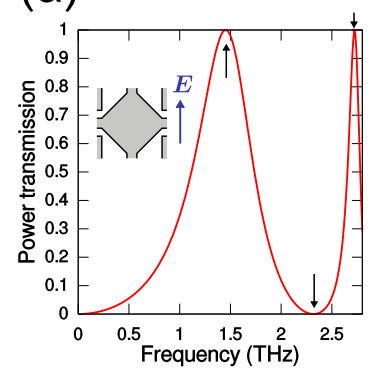

(b)

(c)

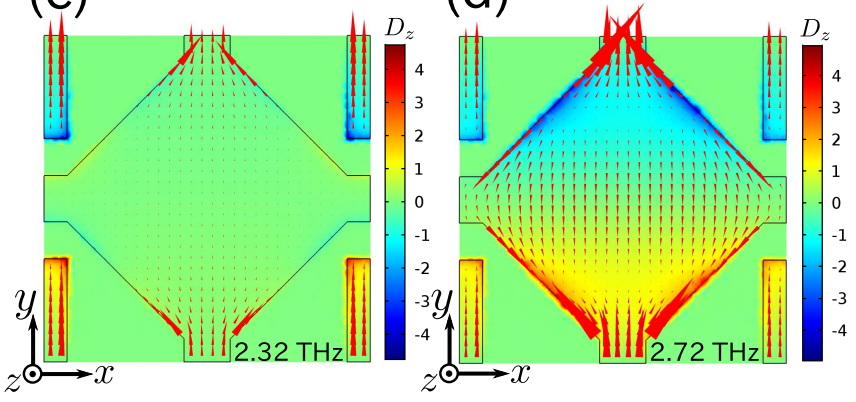

FIG. 6. (a) Transmission spectrum of an on-state checkerboard with dipoles having $a=106 \mu \mathrm{m}, u=w=15 \mu \mathrm{m}$, and $l=67 \mu \mathrm{m}$. Electric displacement $D_{z}$ on the plane $z=a / 1000$, corresponding to the surface charge density, and surface current density on the metasurface at (b) $\omega /(2 \pi)=1.45 \mathrm{THz}$, (c) $2.32 \mathrm{THz}$, and (d) $2.72 \mathrm{THz}$. The phase of $D_{z}$ is shifted by $90^{\circ}$ compared with the surface current.
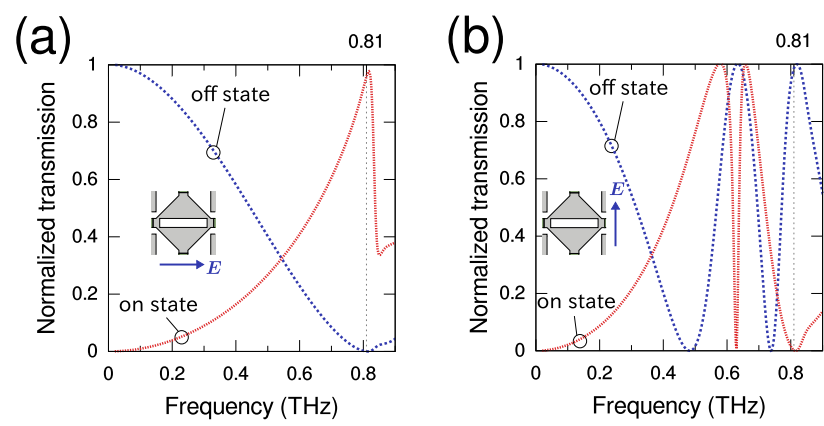

FIG. 7. Calculated normalized power transmission spectra of modified D-checkerboard on a c-cut sapphire substrate at the normal incidence of (a) $x$ - and (b) $y$-polarized plane waves. The geometrical parameters are $a=106 \mu \mathrm{m}, u=w=15 \mu \mathrm{m}$, $g=5 \mu \mathrm{m}, l_{1}=80 \mu \mathrm{m}$, and $l_{2}=69 \mu \mathrm{m}$.

tors. The calculation is performed in the unit cell and periodic boundary conditions are imposed on the sides. The metallic structures are located on $z=0$. A linearlypolarized plane wave is generated from a PML (perfectly matched layer)-backed port with a slit condition on the interior port, and detected by another port with the similar setting. The refractive index of the c-cut sapphire is set to be $n_{x}=n_{y}=n_{\perp}=3.1$ and $n_{z}=n_{\|}=3.4$. The calculated normalized power transmission spectra $|\hat{t}|^{2}$ are shown in Fig. 7 . 
[1] L. Solymar and E. Shamonina, Waves in Metamaterials (Oxford University Press, New York, 2009).

[2] A. V. Kildishev, A. Boltasseva, and V. M. Shalaev, Planar Photonics with Metasurfaces, Science 339, 1232009 (2013)

[3] F. Monticone, N. M. Estakhri, and A. Alù, Full Control of Nanoscale Optical Transmission with a Composite Metascreen, Phys. Rev. Lett. 110, 203903 (2013).

[4] C. Pfeiffer and A. Grbic, Metamaterial Huygens' Surfaces: Tailoring Wave Fronts with Reflectionless Sheets, Phys. Rev. Lett. 110, 197401 (2013)

[5] H. Tao, W. J. Padilla, X. Zhang, and R. D. Averitt, Recent Progress in Electromagnetic Metamaterial Devices for Terahertz Applications, IEEE J. Sel. Topics Quantum Electron. 17, 92 (2011).

[6] H. Booker, SLOT AERIALS AND THEIR RELATION TO COMPLEMENTARY WIRE AERIALS (BABINET'S PRINCIPLE), J. IEE (London), part IIIA 93, 620 (1946).

[7] Rigorously, the sheet impedance is defined as $\tilde{\mathbf{E}}_{\|}(x, y)=$ $Z_{\mathrm{s}}(x, y) \tilde{\mathbf{K}}(x, y)$, for the tangential component of the electric field $\tilde{\mathbf{E}}_{\|}(x, y) \exp (-\mathrm{i} \omega t)+$ c.c. on $z=0$ and surface current density $\tilde{\mathbf{K}}(x, y) \exp (-\mathrm{i} \omega t)+$ c.c. on $z=0$. Here, $\omega$ is an angular frequency and "c.c." represents the complex conjugate operation.

[8] C. E. Baum and B. K. Singaraju, Generalization of Babinet's Principle in Terms of the Combined Field to Include Impedance Loaded Aperture Antennas and Scatterers, Interaction Note No.217 (Air Force Weapons Lab., Kirtland Air Force Base, NM 87117) (1974).

[9] R. C. Compton, J. C. Macfarlane, L. B. Whitbourn, M. M. Blanco, and R. C. McPhedran, Babinet's principle applied to ideal beam-splitters for submillimetre waves, Opt. Acta 31, 515 (1984).

[10] K. Kempa, Percolation effects in the checkerboard Babinet series of metamaterial structures, Phys. Status Solidi Rapid Res. Lett. 4, 218 (2010)

[11] J. D. Edmunds, A. P. Hibbins, J. R. Sambles, and I. J. Youngs, Resonantly inverted microwave transmissivity threshold of metal grids, New J. Phys. 12, 063007 (2010).

[12] K. Takano, F. Miyamaru, K. Akiyama, H. Miyazaki, M. W. Takeda, Y. Abe, Y. Tokuda, H. Ito, and M. Hangyo, Crossover from capacitive to inductive electromagnetic responses in near selfcomplementary metallic checkerboard patterns, Opt. Express 22, 24787 (2014).

[13] S. A. Ramakrishna, P. Mandal, K. Jeyadheepan, N. Shukla, S. Chakrabarti, M. Kadic, S. Enoch, and S. Guenneau, Plasmonic interaction of visible light with gold nanoscale checkerboards, Phys. Rev. B 84, 245424 (2011)

[14] D. González-Ovejero, E. Martini, B. Loiseaux, C. TriponCanseliet, M. J. Mencagli, J. Chazelas, and S. Maci, Basic Properties of Checkerboard Metasurfaces,
IEEE Antennas Wireless Propag. Lett. 14, 406 (2015).

[15] D. González-Ovejero, E. Martini, and S. Maci, Surface Waves Supported by Metasurfaces With Self-Complementary Geometries, IEEE Trans. Antennas Propag. 63, 250 (2015)

[16] B. Tremain, C. J. Durrant, I. E. Carter, A. P. Hibbins, and J. R. Sambles, The Effect of Rotational Disorder on the Microwave Transmission of Checkerboard Metal Square Arrays, Sci. Rep. 5, 16608 (2015)

[17] Y. Nakata, Y. Urade, T. Nakanishi, and M. Kitano, Plane-wave scattering by self-complementary metasurfaces in terms of electromagnetic duality and Babinet's principle, Phys. Rev. B 88, 205138 (2013)

[18] Y. Urade, Y. Nakata, T. Nakanishi, and M. Kitano, Frequency-Independent Response of Self-Complementary Checkerboard Screens, Phys. Rev. Lett. 114, 237401 (2015)

[19] Y. Urade, Y. Nakata, T. Nakanishi, and M. Kitano, Broadband and energy-concentrating terahertz coherent perfect absorber based on a self-complementary metasurface, Opt. Lett. 41, 4472 (2016).

[20] Note that the complex amplitude transmission coefficient $\tilde{t}$ from an incident wave $\check{\mathbf{E}}_{0} \exp \left[\mathrm{i}\left(\mathbf{k}_{0} \cdot \mathbf{x}-\omega t\right)\right]+$ c.c. to a transmitted wave $\check{\mathbf{E}}_{\mathrm{t}} \exp \left[\mathrm{i}\left(\mathbf{k}_{\mathrm{t}} \cdot \mathbf{x}-\omega t\right)\right]+$ c.c. is defined by $\check{\mathbf{E}}_{\mathrm{t}}=\tilde{t} \check{\mathbf{E}}_{0}$. In this paper, the modifier zeroth-order represents the case that $\mathbf{k}_{\mathrm{t}}$ satisfies $\mathcal{P} \mathbf{k}_{\mathrm{t}}=\mathcal{P} \mathbf{k}_{0}$, where $\mathcal{P}:\left(v_{x}, v_{y}, v_{z}\right)^{\mathrm{T}} \mapsto\left(v_{x}, v_{y}, 0\right)^{\mathrm{T}}$ is the projection operator onto $z=0$ ( $\mathrm{T}$ : transpose).

[21] Y. Urade, Y. Nakata, K. Okimura, T. Nakanishi, F. Miyamaru, M. W. Takeda, and M. Kitano, Dynamically Babinet-invertible metasurface: a capacitive-inductive reconfigurable filter for terahertz waves using vanadium-dioxide metal-insulator transition, Opt. Express 24, 4405 (2016)

[22] N. C. J. van der Valk, W. A. M. van der Marel, and P. C. M. Planken, Terahertz polarization imaging, Opt. Lett. 30, 2802 (2005)

[23] S. Katletz, M. Pfleger, H. Pühringer, M. Mikulics, N. Vieweg, O. Peters, B. Scherger, M. Scheller, M. Koch, and K. Wiesauer, Polarization sensitive terahertz imaging: detection of birefringence and optical axis, Opt. Express 20, 23025 (2012).

[24] D. Grischkowsky, S. Keiding, M. van Exter, and C. Fattinger, Far-infrared time-domain spectroscopy with terahertz beams of dielectrics and semiconductors, J. Opt. Soc. Am. B 7, 2006 (1990)

[25] K. Okimura, Y. Sasakawa, and Y. Nihei, X-ray Diffraction Study of Electric Field-Induced MetalInsulator Transition of Vanadium Dioxide Film on Sapphire Substrate, Jpn. J. Appl. Phys. 45, 9200 (2006)

[26] J. Nag and R. F. Haglund Jr, Synthesis of vanadium dioxide thin films and nanoparticles, J. Phys.: Condens. Matter 20, 264016 (2008).

[27] A. E. Miroshnichenko, S. Flach, and Y. S. Kivshar, Fano resonances in nanoscale structures, Rev. Mod. Phys. 82, 2257 (2010) 